\title{
Effect of Neoadjuvant Chemoradiotherapy on Lymph Node Micrometastases in Thoracic Esophageal Cancer
}

\author{
MASAYUKI YANAGI ${ }^{1,2^{*}}$, KEN SASAKI $^{1 *}$, YASUTO UCHIKADO ${ }^{1}$, ITARU OMOTO $^{1}$, \\ TAKAAKI ARIGAMI ${ }^{1}$, HIROSHI KURAHARA ${ }^{1}$, YOSHIKAZU UENOSONO ${ }^{1}$, \\ MASAHIKO SAKODA ${ }^{1}$, KOSEI MAEMURA $^{1}$ and SHOJI NATSUGOE ${ }^{1}$ \\ ${ }^{1}$ Department of Digestive Surgery, Breast and Thyroid Surgery, \\ Graduate School of Medical and Dental Sciences, Kagoshima University, Kagoshima, Japan; \\ ${ }^{2}$ Department of Gastoenterogical Surgery, Kagoshima City Hospital, Kagoshima, Japan
}

\begin{abstract}
Aim: The purpose of this study was to clarify the effect of neoadjuvant chemoradiotherapy ( $n C R T)$ on lymph node micrometastasis (LNM) in esophageal squamous cell carcinoma (ESCC). Patients and Methods: The therapeutic efficacy of $n C R T$ was analyzed in 41 ESCC patients randomized to the Surgery group $(n=21)$ and the $n C R T$ group $(n=20)$. Lymph node specimens from patients were classified into two categories, micrometastasis (MM) and tumor cell microinvolvement (MI), after immunohistochemical evaluation. Results: The incidence rates of patients presenting MM with or without MI or MI alone in the Surgery group were significantly higher than those in the nCRT group. The 10-year survival rate of 15 patients with simultaneous histological metastasis (HM) and LNM was significantly lower than that in the 26 patients without LNM. Within the nCRT group, the 10-year survival rates of patients with versus those without HM were not significantly different; however, the 10-year survival rate of the 5 patients with simultaneous HM and LNM was significantly lower than that of the 15 patients without LNM. Conclusion: ESCC patients with LNM may benefit from $n C R T$, and evaluation of the simultaneous presence of HM and LNM may facilitate accurate prediction of survival in ESCC patients.
\end{abstract}

Treatment strategy of esophageal cancer is difficult to define, and it has been reported that not only staging of lymph node

*These Authors contributed equally to this work.

Correspondence to: Ken Sasaki, MD, Ph.D., Department of Digestive Surgery, Breast and Thyroid Surgery, Graduate School of Medical and Dental Sciences, Kagoshima University, Sakuragaoka 8-35-1, Kagoshima-shi, Kagoshima 890-8520, Japan. Tel: +81 992755361, Fax: +81 992657426, e-mail: k-sasaki@m.kufm.kagoshima-u.ac.jp

Key Words: Lymph node metastasis, lymph node micrometastasis, cytokeratin, neoadjuvant chemoradiotherapy, esophageal cancer, randomized controlled trial. metastasis, but also the number of lymph node metastases are important prognostic factors for guiding disease treatment (13 ). Based on evidence from three randomized controlled trials (RCTs), preoperative chemotherapy or chemoradiotherapy (CRT) has been performed to improve esophageal cancer treatment outcomes (4-6). The aim of this preoperative treatment is to control lymph node micrometastasis (LNM) and extra-nodal cancer cell invasion in connective tissue. In previous reports, investigators have examined lymph node tissue specimens from node-negative esophageal cancer patients, using cytokeratin immunohistochemistry (IHC), and have shown that micrometastases were associated with poor prognosis after curative resection in the absence of preoperative treatment (7-9). However, very few studies have focused on the relationship between neoadjuvant CRT (nCRT) and LNM in esophageal cancer patients.

The purpose of the present study was to clarify the therapeutic efficacy of neoadjuvant chemoradiotherapy (nCRT) on LNM and the impact of LNM on long-term survival (>10 years) in esophageal squamous cell carcinoma (ESCC).

\section{Patients and Methods}

Study group. A total of 53 patients with ESCC were eligible during the study period; 8 refused randomization and elected to undergo upfront surgery. Thus, 45 patients were enrolled in this RCT between 1997 and 2001. Twenty-three patients were assigned to the Surgery group and 22 to the nCRT group, while 2 patients with non-completion of LNM evaluation in the Surgery group and 2 patients with appearance of distant metastasis after nCRT were excluded (Figure 1). The eligibility criteria had been described in our previous report (10). In brief, criteria were as follows: 1) squamous cell carcinoma of the thoracic esophagus excluding T4 disease; 2 ) resectable disease; 3 ) age 70 years or younger without synchronous or metachronous cancer in other organs; 4) Karnofsky performance status $\geq 90 \%$; and 5) sufficient organ function. Esophagogastro-duodenoscopy, esophagography, endoscopic ultrasonography, ultrasonography, and computed tomography were required, and bronchoscopy and bronchoscopic ultrasonography were 


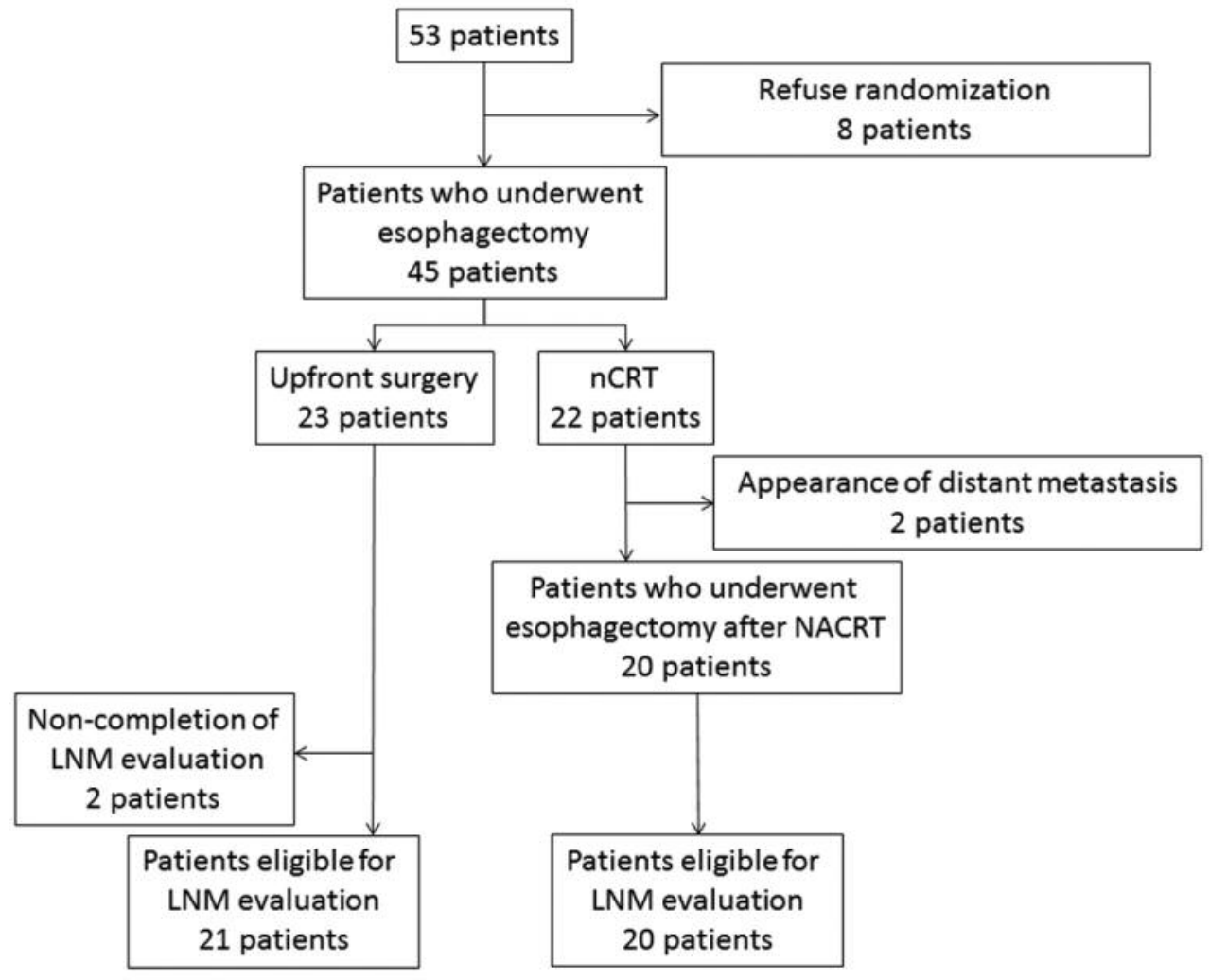

Figure 1. Consolidated standards of reporting trials (CONSORT) flow diagram presents the progress of all participants through the study. $n C R T$, Neoadjuvant chemoradiotherapy; LNM, lymph node micrometastasis.

recommended for clinical staging. Importantly, lymph node metastasis was evaluated by endoscopic ultrasonography and ultrasonography based on previously described criteria (11). Written informed consent was obtained from each patient. After agreement, patients were randomly assigned to one of two groups: the Surgery group, treated with surgery alone, and the nCRT group, treated with nCRT followed by surgery. Tumors were classified using the UICC tumor-nodemetastasis (TNM) classification system (12). All patients were followed up every 3 months for a year and then every 3 to 6 months. The median follow-up time after surgery was 90 months (range=18-170). The clinicopathological characteristics of patients at diagnosis are shown in Table I. All M1 cases had supraclavicular lymph node metastases. Institutional review board of Kagoshima University approved this protocol and retrospective study (approval number 25-39).

Chemoradiotherapy. A total radiation dose of $40 \mathrm{~Gy}$ (2 Gy/day for 5 days/week) was applied to the mediastinum and neck. Concurrent intravenous chemotherapy consisting of cisplatin $(7 \mathrm{mg}$ over $2 \mathrm{~h}$ ) and 5-fluorouracil (5-FU; $350 \mathrm{mg}$ over $24 \mathrm{~h}$ ) was administered.

The histopathological response to CRT was classified in accordance with the criteria of the Japanese Society for Esophageal Diseases. Briefly, the degree of viability of the residual tumor cells was evaluated as follows: Grade 0 , no change; Grade 1, more than one third of the residual tumor cells were viable; Grade 2, less than one third of the residual tumor cells were viable; and Grade 3, histological fibrosis with or without granulomatous changes, and no
Table I. Clinical characteristics of patients at diagnosis.

\begin{tabular}{lccc}
\hline Category & $\begin{array}{c}\text { Surgery group } \\
\mathrm{n}=23\end{array}$ & $\begin{array}{c}\mathrm{nCRT} \text { group } \\
\mathrm{n}=22\end{array}$ & $p$-Value \\
\hline T category & 1 & 2 & 0.968 \\
cT2 & & 20 & \\
cT3 & 22 & 3 & 0.958 \\
N category & & 19 & \\
cN0 & 2 & & 0.50 \\
cN1 & 21 & 19 & \\
M category & 17 & 3 & \\
cM0 & 6 & & 0.88 \\
cM1 & & 5 & \\
cStage & 4 & 11 & \\
II & 13 & 6 & \\
III & 6 & & \\
IV & & & \\
\hline
\end{tabular}

c, Clinical; M, metastases; N, lymph nodes; nCRT, neoadjuvant chemoradiotherapy; $\mathrm{T}$, tumor.

viable tumor cells were observed (13). CRT was defined as effective if patients had Grade 2 or 3 histopathological responses (responders), and conversely, as ineffective if patients had Grade 0 or 1 histopathological responses (non-responders). 

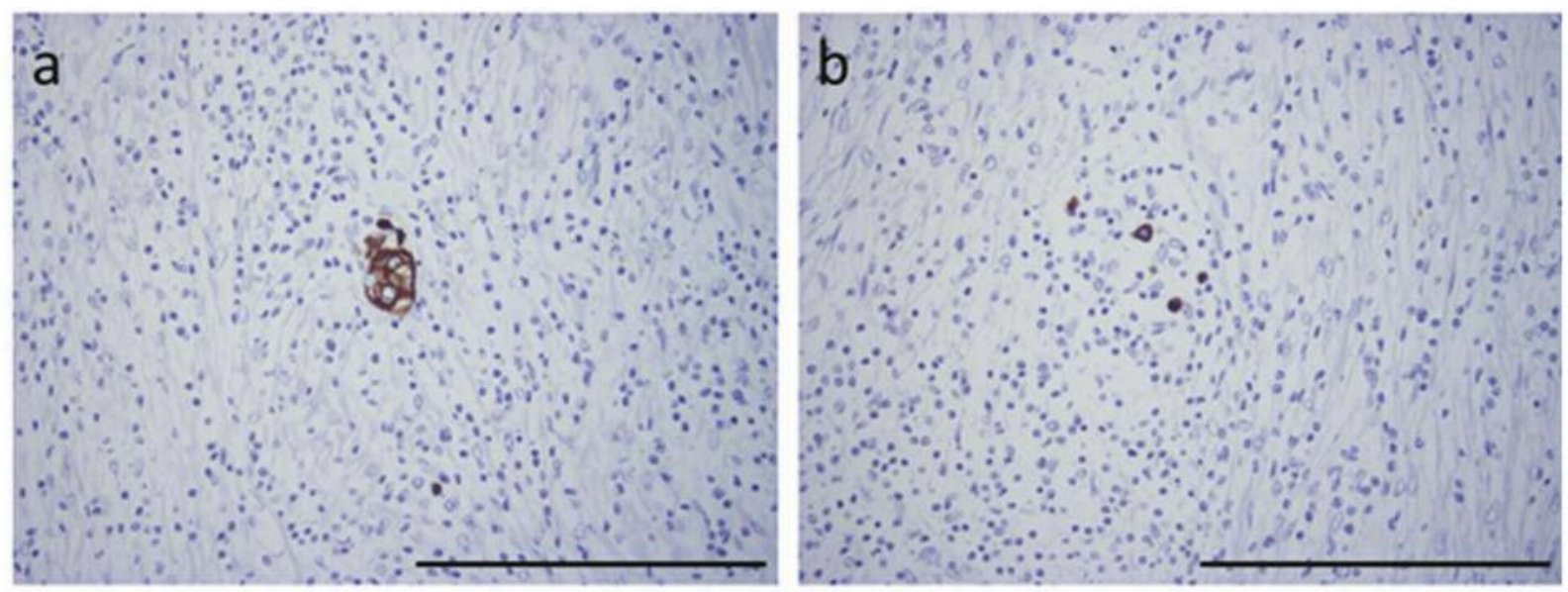

Figure 2. Immunohistochemical detection of micrometastasis (MM) and microinvolvement (MI). Lymph node sections from ESCC tissue were stained with the antibody cocktail AE1/AE3 (original magnification, x400). An example of MM (a) and MI (b). Scale bar=200 $\mu m,(a)$ and $(b)$.

IHC. All lymph nodes were excised at esophagectomy, and all samples were formalin-fixed and paraffin-embedded. Each lymph node was sliced at its maximum diameter to obtain a maximal section area, and was stained with hematoxylin and eosin (HE) for routine histological examination. One additional section was stained by IHC using AE1/AE3 (20:1 mixture of AE1 to AE3; Boehringer, Mannheim, Germany), a monoclonal antibody cocktail that is reactive with a broad spectrum of human cytokeratins (CKs) (14). Tissue sections were deparaffinized in xylene and rehydrated using a series of graded ethanol solutions. Antigen retrieval was performed by autoclaving in $10 \mathrm{mM}$ citrate buffer solution for 6 $\min$. Sections were incubated with the anti-CK monoclonal antibody at a 1:100 dilution, followed by avidin-biotin-peroxidase (Nichirei Biosciences Inc., Tokyo, Japan) staining. The immunohistochemical assessment was limited to one slide per lymph node to simulate routine histological procedures. The primary antibodies were replaced with phosphate-buffered saline for negative controls. Normal esophageal epithelium and primary cancers were used as positive controls for $\mathrm{CK}$ and were consistently positive.

Histological evaluation. Lymph node metastases larger than 0.5 $\mathrm{mm}$ in their greatest dimension, which were detected by HEstaining, were designated as histological metastases (HM). Tumor involvement of lymph nodes detected by IHC was divided into 2 categories as described in our previous report: i) micrometastasis $\mathrm{MM}$, a cluster of tumor cells smaller than $0.5 \mathrm{~mm}$ in greatest dimension (Figure 2A); and ii) microinvolvement (MI), a single cell metastasis (Figure 2B) (15). Immunohistochemical results were examined by 2 independent and blinded observers (M.Y. and S.N.). Each lymph node was evaluated in the whole area of the section.

Statistical analysis. Statistical analysis of differences between the two groups was carried out using the Chi-squared test. Data are shown as mean \pm standard deviation. A $p$-value $<0.05$ was considered to be statistically significant. Survival curves were calculated by the Kaplan-Meier method and were compared using the log-rank test.

\section{Results}

Detection of preoperative lymph node metastasis by imaging . Preoperatively, lymph node metastasis was diagnosed by endoscopic ultrasonography and ultrasonography in 17 of 20 patients in the nCRT group and 21 of 23 patients in the Surgery group. Patients in the Surgery and nCRT groups had

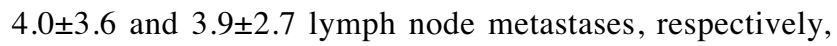
before surgery. There was no statistically significant difference between groups.

Adverse events and histological analysis of primary tumor and nodal metastases. As described in our previous report, adverse events involving gastrointestinal and hematological toxicity were common. Grade 3 adverse events included anorexia $(n=1)$, leukopenia $(n=3)$, anemia $(n=1)$, and platelet count decreased $(n=1)$. No serious complications requiring cessation of CRT occurred. Histological analysis of primary tumors revealed that 10,7, and 3 patients had Grade 1, 2, and 3 tumors, respectively. In the nCRT group, although all primary tumors $(n=20)$ were preoperatively diagnosed as cT2 or cT3, pT0 and pT1 tumors were histologically diagnosed in 3 patients each. Lymph node metastasis including distant nodal involvement (M1 TNM staging) was detected by routine histological examination (HE) in 15 of 21 (71.4\%) patients in the Surgery group and 12 of $20(60.0 \%)$ patients in the nCRT group. Of the 1,078 nodes obtained by lymph node dissection in 21 patients in the Surgery group, 132 nodes $(12.2 \%)$ contained cancer cells. Of the 20 patients in the nCRT group, lymph node metastasis was observed in 41 of 663 nodes $(6.2 \%)$ by routine histological examination. However, differences on lymph node metastasis rates and the number of lymph node metastases between groups were not 
Table II. Clinicopathological characteristics of patients at diagnosis.

\begin{tabular}{lccc}
\hline Category & $\begin{array}{c}\text { Surgery group } \\
\mathrm{n}=21\end{array}$ & $\begin{array}{c}\mathrm{nCRT} \text { group } \\
\mathrm{n}=20\end{array}$ & $p$-Value \\
\hline Age, mean (range) & $60.0(38-70)$ & $61.5(42-69)$ & 0.552 \\
Gender ratio & $20: 1$ & $20: 0$ & 0.243 \\
T category & & & $\mathbf{0 . 0 0 3}$ \\
pT0 & 0 & 3 & \\
pT1 & 0 & 3 & \\
pT2 & 3 & 0 & \\
pT3 & 18 & 12 & \\
pT4 & 0 & 2 & \\
N category & & & \\
pN0 & 6 & 8 & \\
pN1 & 15 & 12 & \\
M category & & & \\
pM0 & 15 & 14 & \\
pM1 & 6 & 6 & \\
pStage & & & \\
0 & 0 & 3 & \\
I & 2 & 0.185 \\
II & 5 & 7 & \\
III & 8 & 6 & \\
IV & 6 & 4 & \\
Lymphatic invasion & & 11 & \\
$(-)$ & 3 & 9 & \\
$(+)$ & 18 & & \\
Venous invasion & 5 & & \\
$(-)$ & 16 & & \\
$(+)$ & & & \\
\hline
\end{tabular}

Values in bold indicate significance. c, Clinical; M, metastases; N, lymph nodes; nCRT, neoadjuvant chemoradiotherapy; T, tumor.

statistically significant. The frequencies of lymphatic and venous invasion were significantly lower in the nCRT group than in the Surgery group (Table II) (10).

Immunohistochemical evaluation of lymph node micrometastasis. Immunohistochemical examination revealed 28 nodes with MM and 9 nodes with $\mathrm{MI}$ in the Surgery group, totaling $3.4 \%$ (37/1078) of resected nodes. In contrast, the numbers of nodes with MM and MI in the nCRT group were 4 and 11 , respectively, totaling $2.3 \%$ (15/663). The incidence of patients presenting MM with or without MI in the Surgery group (76.2\%) was significantly higher than that in the nCRT group $(30.0 \% ; p=0.0025)$. Furthermore, the incidence of patients with MI alone in the Surgery group $(19.0 \%)$ was significantly higher than that in the nCRT group ( $0 \% ; p=0.0164)$ (Table III). In the nCRT group, the incidence of patients presenting MM with or without MI among responders was considerably lower than among non-responders, although the difference was not statistically significant ( $p=0.083$, Table IV). Analysis of the relationship between MM with or without MI and TNM
Table III. Incidence of micrometastasis and/or tumor cell microinvolvement in all patients.

\begin{tabular}{lccc}
\hline Category & $\begin{array}{c}\text { Surgery group } \\
\mathrm{n}=21\end{array}$ & $\begin{array}{c}\mathrm{nCRT} \text { group } \\
\mathrm{n}=20\end{array}$ & $p$-Value \\
& & & 0.441 \\
Lymph node metastasis by HE & 15 & 12 & \\
$\quad$ Yes & 6 & 8 & $\mathbf{0 . 0 0 3}$ \\
No & & & \\
MM \pm MI & 16 & 6 & \\
$\quad$ Yes & 5 & 14 & $\mathbf{0 . 0 1 6}$ \\
No & & & \\
MI alone & 4 & 0 & \\
Yes & 17 & 20 & \\
No & 17 & &
\end{tabular}

Values in bold indicate significance. HE, Hematoxylin and eosin; nCRT, neoadjuvant chemoradiotherapy; $\mathrm{MM} \pm \mathrm{MI}$, micrometastasis with or without microinvolvement.

Table IV. Incidence of micrometastasis and/or tumor cell microinvolvement in the neoadjuvant chemoradiotherapy group.

\begin{tabular}{|c|c|c|c|}
\hline \multirow[t]{2}{*}{ Category } & \multicolumn{2}{|c|}{ nCRT group } & \multirow[b]{2}{*}{$p$-Value } \\
\hline & $\begin{array}{c}\text { Grade } 0 / 1 \\
\mathrm{n}=11\end{array}$ & $\begin{array}{c}\text { Grade } 2 / 3 \\
n=9\end{array}$ & \\
\hline Lymph node metastasis by HE & & & 0.927 \\
\hline Yes & 6 & 6 & \\
\hline No & 5 & 3 & \\
\hline $\mathrm{MM} \pm \mathrm{MI}$ & & & 0.083 \\
\hline Yes & 5 & 1 & \\
\hline No & 6 & 8 & \\
\hline MI alone & & & N/A \\
\hline Yes & 0 & 0 & \\
\hline No & 11 & 9 & \\
\hline
\end{tabular}

HE, Hematoxylin and eosin; nCRT, neoadjuvant chemoradiotherapy; $\mathrm{MM} \pm \mathrm{MI}$, micrometastasis with or without microinvolvement.

category revealed that patients with more advanced-stage disease were more likely to have MM with or without MI in both the Surgery and nCRT groups (Table V).

Clinical outcomes. Although the 10-year survival rate was higher in the nCRT group compared to the Surgery group, this difference did not reach statistical significance $(48.8 \%$ vs. $19.1 \% ; p=0.172$; Figure 3 ). Within the nCRT group, the 10-year survival rates of responders and non-responders were $63.5 \%$ and $36.4 \%$, respectively, though not statistically significant ( $p=0.133$; Figure 4$)$. In terms of lymph node metastasis status regardless of preoperative treatment, the 10year survival rate of patients with HM ( $n=27)$ was lower than 


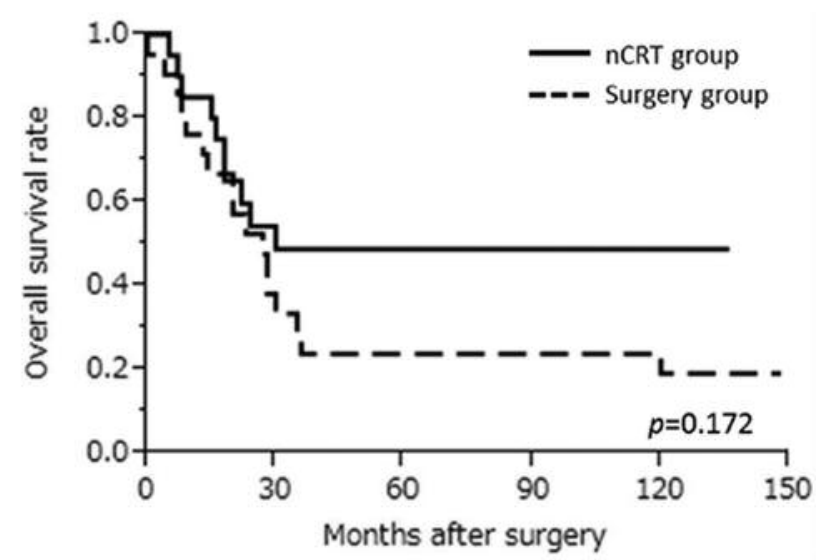

Figure 3. Overall survival rates in the Surgery and $n C R T$ group. The 10 -year survival rate was $19.1 \%$ in the Surgery group and $48.8 \%$ in the $n C R T$ group; this difference was not statistically significant $(p=0.172)$. $n C R T$, Neoadjuvant chemoradiotherapy.

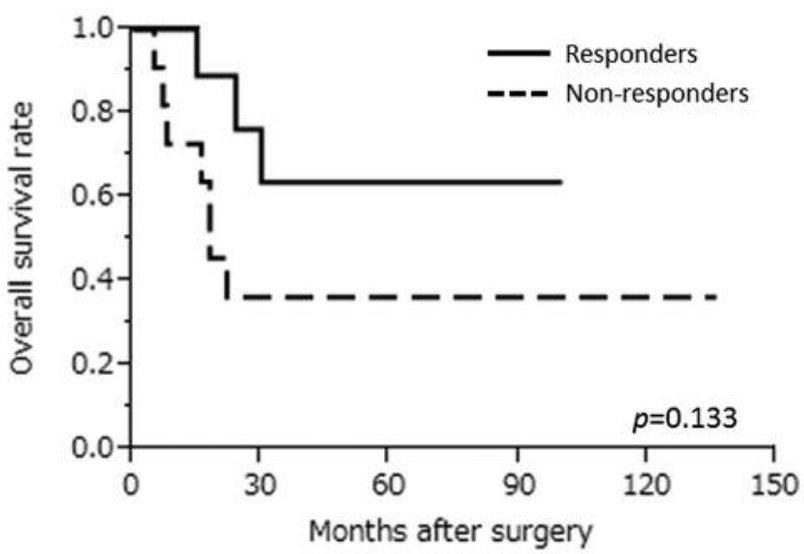

Figure 4. Overall survival rates within the $n C R T$ group according to histological analysis. The 10-year survival rate of responders and nonresponders was $63.5 \%$ and $36.4 \%$, respectively; this difference was not statistically significant ( $p=0.133$ ). $n C R T$, Neoadjuvant chemoradiotherapy.

Table V. TNM category and micrometastasis and/or tumor cell microinvolvement.

\begin{tabular}{|c|c|c|c|c|c|c|c|c|}
\hline \multirow[t]{2}{*}{ Category } & \multicolumn{4}{|c|}{ Surgery group $(n=21)$} & \multicolumn{4}{|c|}{ nCRT group $(n=20)$} \\
\hline & Case & Positive & $\mathrm{MM} \pm \mathrm{MI}$ & MI alone & Case & Positive & $\mathrm{MM} \pm \mathrm{MI}$ & MI alone \\
\hline \multicolumn{9}{|l|}{$\mathrm{T}$ category } \\
\hline pT0 & 0 & 0 & 0 & 0 & 3 & 1 & 1 & 0 \\
\hline pT1 & 0 & 0 & 0 & 0 & 3 & 0 & 0 & 0 \\
\hline pT2 & 3 & 2 & 0 & 2 & 3 & 0 & 0 & 0 \\
\hline pT3 & 18 & 14 & 12 & 2 & 9 & 4 & 4 & 0 \\
\hline pT4 & 0 & 0 & 0 & 0 & 2 & 1 & 1 & 0 \\
\hline \multicolumn{9}{|l|}{$\mathrm{N}$ category } \\
\hline $\mathrm{pNO}$ & 6 & 3 & 0 & 3 & 8 & 2 & 2 & 0 \\
\hline $\mathrm{pN} 1$ & 15 & 13 & 12 & 1 & 12 & 4 & 4 & 0 \\
\hline \multicolumn{9}{|l|}{ M category } \\
\hline $\mathrm{pM} 0$ & 15 & 10 & 7 & 3 & 14 & 4 & 4 & 0 \\
\hline pM1 & 6 & 6 & 5 & 1 & 6 & 2 & 2 & 0 \\
\hline
\end{tabular}

HE, Hematoxylin and eosin; MM \pm MI, micrometastasis with or without microinvolvement; $\mathrm{nCRT}$, neoadjuvant chemoradiotherapy.

that of patients without HM $(\mathrm{n}=14)(23.7 \%$ vs. $42.9 \%$; $p=0.063$; Figure $5 \mathrm{~A})$. The 10 -year survival rate of the 15 patients with simultaneous HM and LNM was significantly lower than that of the 26 patients without LNM $(5.9 \% \mathrm{vs}$. $45.9 \% ; p=0.001$; Figure 5B). Within the nCRT group, the 10 -year survival rate was increased in patients without HM $(\mathrm{n}=8)$ compared to patients with HM $(\mathrm{n}=12)$; this trend was not statistically significant $(62.5 \%$ vs. $38.9 \% ; p=0.290$; Figure 5C). However, the 10-year survival rate of the 5 patients with simultaneous HM and LNM was $0 \%$, while that of the 15 patients without LNM was $61.4 \%$; the survival difference between these groups was statistically significant $(p=0.011$; Figure 5D).

\section{Discussion}

The clinical aspects of LNM in gastrointestinal tract cancers have been previously discussed in detail (16), and the presence of LNM is correlated with poor prognosis in ESCC patients $(7,8)$. Currently, nCRT followed by surgery has been performed as the standard treatment for resectable esophageal cancer, based on a large-scale Dutch RCT (the 
a

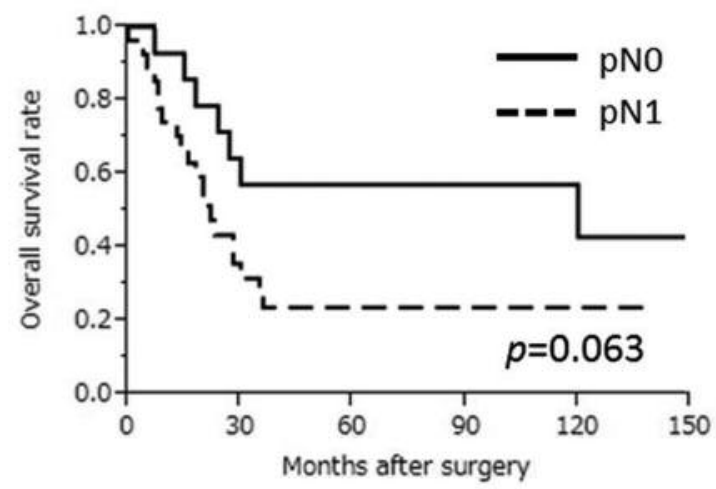

b

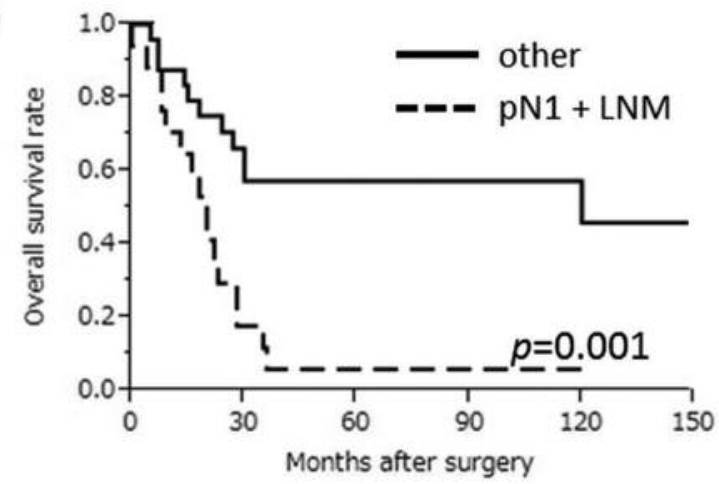

C

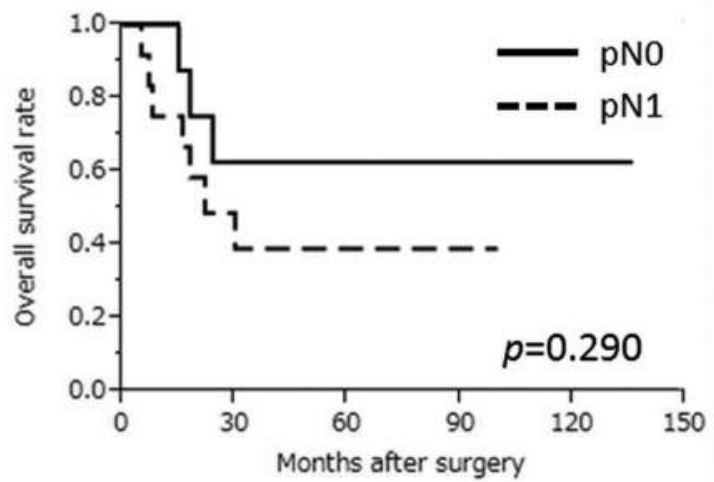

d

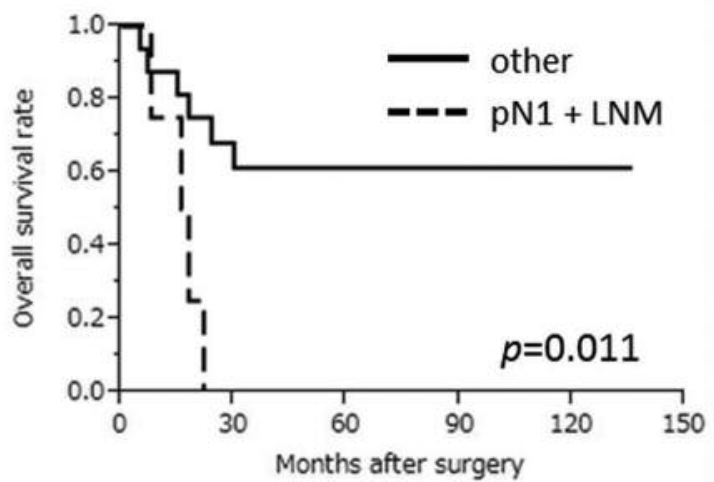

Figure 5. Overall survival according to nodal metastasis status. Overall survival according to (A) the presence or absence of nodal metastasis detected by routine histological examination using HE, (B) simultaneous histological nodal metastasis and immunohistochemical nodal metastasis, $(C)$ the presence or absence of nodal metastasis in patients treated with $n C R T$, and (D) simultaneous histological nodal metastasis and immunohistochemical nodal metastasis in patients treated with nCRT. HE, Hematoxylin and eosin; pNO, absence of nodal metastasis; pN1, presence of nodal metastasis; LNM, lymph node micrometastasis; $n C R T$, neoadjuvant chemoradiotherapy.

CROSS trial) (5). Although one of the potential benefits of nCRT is considered to be elimination of LNM, no fundamental data confirm this theory. To the best of our knowledge, this is the first prospective study to demonstrate the therapeutic efficacy of nCRT against LNM, including the impact on long-term survival ( $>10$ years) in ESCC patients randomized to nCRT or surgery.

In the present study the survival rate was higher in the nCRT group than in the Surgery group, which is consistent with the results of a large-scale RCT of nCRT for esophageal cancer (5). Though, due to the small sample size of this study, this difference was not statistically significant. Similarly, no statistically significant efficacy was observed for the nCRT against lymph node metastasis. However, the incidence rates of MM with or without MI and MI alone in the nCRT group were significantly lower than those in the Surgery group, indicating that patients presenting MM with or without MI and MI alone might benefit from nCRT. These results are consistent with the retrospective study of Wang et $a l$, in which significant reduction of LNM was obtained after nCRT in node-negative esophageal adenocarcinoma patients (17). Whether LNM progresses to overt metastasis or regresses spontaneously due to immune system activity cannot be determined. Yokoyama et al. used a mouse model and demonstrated that isolated tumor cells (ITCs) regressed spontaneously through natural killer cell-mediated antitumor activity following resection of primary subcutaneous tumors, whereas MM continued to proliferate and develop into gross disease. Thus, MM and ITCs appear to behave differently after surgical intervention (18). For the reasons distributed above, we categorized two types of LNM, MM and MI. In contrast to nCRT, the efficacy of neoadjuvant chemotherapy (nCT) for LNM in ESCC has yet to be demonstrated. Matsuyama et al. reported that $\mathrm{nCT}$ may eradicate LNM (19); however, our previous prospective study showed no significant differences between patients who received nCT versus surgery alone with regard to the presence of MM with or without MI, except for some patients with just MI (15). 
The results of our present and previous studies suggest the possibility that nCRT may be more effective than $\mathrm{nCT}$ in reducing LNM.

Moreover, in the present study there was evaluated only the significance of simultaneous HM and LNM, because the number of patients without HM was small in each group. The presence of HM was not a significant prognostic factor, neither in all patients nor in the nCRT group, whereas the presence of simultaneous HM and LNM was a significant prognostic factor in all patients as well as in the nCRT group. Most previous studies that evaluated the prognostic impact of LNM in esophageal cancer limited their investigation to node-negative esophageal cancer treated by surgery alone (20); therefore, the clinical aspect of LNM remains uncertain in patients with node-positive esophageal cancer or patients who undergo preoperative treatment. The present results are in agreement with those of Komukai et al. (21) and Kinjo et al. (22) in that evaluation of the simultaneous presence of HM and LNM may facilitate highly accurate prediction of survival in esophageal cancer. This evidence supports the value of preoperative control of LNM not only in nodenegative ESCC patients but also in node-positive patients.

This is the first prospective study to show that nCRT can successfully reduce LNM, resulting in favorable long-term survival. One of the limitations of this study was that the sample size was relatively small. Furthermore, limited information was available regarding the effects of nCRT in clinical samples of LNM, and it is impossible to evaluate whether LNMs were eradicated by nCRT in clinical samples. Some previous studies evaluated cytokeratin deposit (CD), which was defined as a clustering of a few or more particles that expressed cytokeratin and were similar in size to esophageal cancer cells, but did not have nuclei, in LNM treated by nCT in ESCC $(19,23)$. However, it is not certain whether CDs arise from dead or dying cancer cells. Therefore, further consideration is needed, preferably in a larger, prospective dataset. In conclusion, our study demonstrated that LNM in patients with ESCC may benefit from nCRT, and evaluation of the simultaneous presence of HM and LNM may facilitate accurate prediction of survival in ESCC patients treated with nCRT.

\section{Ethical Statement}

All procedures followed were in accordance with the ethical standards of the responsible committee on human experimentation (institutional and national) and with the Helsinki Declaration of 1964 and later versions. Informed consent or substitute for it was obtained from all patients for being included in the study.

\section{Conflicts of Interest}

All Authors declare no financial or commercial conflict of interest in regard with this study.

\section{Acknowledgements}

This work was supported by JSPS KAKENHI Grant Number 17K10706. The Authors gratefully acknowledge the work of our laboratory's members.

\section{References}

1 Natsugoe S, Yoshinaka H, Shimada M, Sakamoto F, Morinaga T, Nakano S, Kusano C, Baba M, Takao S and Aikou T: Number of lymph node metastases determined by presurgical ultrasound and endoscopic ultrasound is related to prognosis in patients with esophageal carcinoma. Ann Surg 234: 613-618, 2001.

2 Okumura H, Uchikado Y, Matsumoto M, Owaki T, Kita Y, Omoto I, Sasaki K, Sakurai T, Setoyama T, Nabeki B, Matsushita D, Ishigami S, Hiraki Y, Nakajo M and Natsugoe S: Prognostic factors in esophageal squamous cell carcinoma patients treated with neoadjuvant chemoradiation therapy. Int $\mathrm{J}$ Clin Oncol 18: 329-334, 2013.

3 Roder JD, Busch R, Stein HJ, Fink U and Siewert JR: Ratio of invaded to removed lymph nodes as a predictor of survival in squamous cell carcinoma of the oesophagus. Br J Surg 81: 410413, 1994.

4 Cunningham D, Allum WH, Stenning SP, Thompson JN, Van de Velde CJ, Nicolson M, Scarffe JH, Lofts FJ, Falk SJ, Iveson TJ, Smith DB, Langley RE, Verma M, Weeden S, Chua YJ and Participants MT: Perioperative chemotherapy versus surgery alone for resectable gastroesophageal cancer. N Engl J Med 355: 11-20, 2006.

5 Shapiro J, van Lanschot JJ, Hulshof MC, van Hagen P, van Berge Henegouwen MI, Wijnhoven BP, van Laarhoven HW, Nieuwenhuijzen GA, Hospers GA, Bonenkamp JJ, Cuesta MA, Blaisse RJ, Busch OR, Ten Kate FJ, Creemers GJ, Punt CJ, Plukker JT, Verheul HM, Bilgen EJ, van Dekken H, van der Sangen MJ, Rozema T, Biermann K, Beukema JC, Piet AH, van Rij CM, Reinders JG, Tilanus HW, Steyerberg EW, van der Gaast A and group Cs: Neoadjuvant chemoradiotherapy plus surgery versus surgery alone for oesophageal or junctional cancer (CROSS): long-term results of a randomised controlled trial. Lancet Oncol 16: 1090-1098, 2015.

6 Ando N, Kato H, Igaki H, Shinoda M, Ozawa S, Shimizu H, Nakamura T, Yabusaki H, Aoyama N, Kurita A, Ikeda K, Kanda T, Tsujinaka T, Nakamura $\mathrm{K}$ and Fukuda $\mathrm{H}$ : A randomized trial comparing postoperative adjuvant chemotherapy with cisplatin and 5-fluorouracil versus preoperative chemotherapy for localized advanced squamous cell carcinoma of the thoracic esophagus (JCOG9907). Ann Surg Oncol 19: 68-74, 2012.

7 Natsugoe S, Mueller J, Stein HJ, Feith M, Hofler H and Siewert JR: Micrometastasis and tumor cell microinvolvement of lymph nodes from esophageal squamous cell carcinoma: frequency, associated tumor characteristics, and impact on prognosis. Cancer 83: 858-866, 1998.

8 Izbicki JR, Hosch SB, Pichlmeier U, Rehders A, Busch C, Niendorf A, Passlick B, Broelsch CE and Pantel K: Prognostic value of immunohistochemically identifiable tumor cells in lymph nodes of patients with completely resected esophageal cancer. N Engl J Med 337: 1188-1194, 1997. 
9 Matsumoto M, Natsugoe S, Nakashima S, Sakamoto F, Okumura H, Sakita H, Baba M, Takao S and Aikou T: Clinical significance of lymph node micrometastasis of pN0 esophageal squamous cell carcinoma. Cancer Lett 153: 189-197, 2000.

10 Natsugoe S, Okumura H, Matsumoto M, Uchikado Y, Setoyama $\mathrm{T}$, Yokomakura $\mathrm{N}$, Ishigami $\mathrm{S}$, Owaki $\mathrm{T}$ and Aikou $\mathrm{T}$ : Randomized controlled study on preoperative chemoradiotherapy followed by surgery versus surgery alone for esophageal squamous cell cancer in a single institution. Dis Esophagus 19: 468-472, 2006.

11 Sakamoto F, Natsugoe S, Yoshinaka H, Shimada M, Owaki T, Nakano S, Baba M and Aikou T: Endosonographic detection of mediastinal lymph node metastasis in superficial carcinoma of the esophagus: assessment by type classification and histogram. J Gastroenterol 39: 7-13, 2004.

12 Sobin L H WCH: TNM Classification of Malignant Tumours, 6th edition: New York: Wiley-Liss 2002.

13 Japanese Society for Esophageal Diseases. Guidelines for the Clinical and Pathological Studies on Carcinoma of the Esophagus. Tokyo: Kanehara, 1999.

14 Listrom MB and Dalton LW: Comparison of keratin monoclonal antibodies MAK-6, AE1:AE3, and CAM-5.2. Am J Clin Pathol 88: 297-301, 1987.

15 Natsugoe S, Matsumoto $M$, Nakashima S, Okumura $H$, Miyazono F, Kijima F, Ishigami S, Aridome K, Kusano C, Baba M, Takao S and Aikou T: Effect of neoadjuvant chemotherapy for lymph node micrometastasis and tumor cell microinvolvement in the patients with esophageal carcinoma. Cancer Lett 159: 119-125, 2000.

16 Natsugoe S, Arigami T, Uenosono Y, Yanagita S, Nakajo A, Matsumoto M, Okumura H, Kijima Y, Sakoda M, Mataki Y, Uchikado Y, Mori S, Maemura K and Ishigami S: Lymph node micrometastasis in gastrointestinal tract cancer - a clinical aspect. Int J Clin Oncol 18: 752-761, 2013.

17 Wang D, Smit JK, Zwaan E, Muijs CT, Groen H, Hollema H and Plukker JT: Neoadjuvant therapy reduces the incidence of nodal micrometastases in esophageal adenocarcinoma. Am J Surg 206: 732-738, 2013.
18 Yokoyama H, Nakanishi H, Kodera Y, Ikehara Y, Ohashi N, Ito Y, Koike M, Fujiwara M, Tatematsu M and Nakao A: Biological significance of isolated tumor cells and micrometastasis in lymph nodes evaluated using a green fluorescent protein-tagged human gastric cancer cell line. Clin Cancer Res 12: 361-368, 2006.

19 Matsuyama J, Doki Y, Yasuda T, Miyata H, Fujiwara Y, Takiguchi S, Yamasaki M, Makari Y, Matsuura N, Mano M and Monden M: The effect of neoadjuvant chemotherapy on lymph node micrometastases in squamous cell carcinomas of the thoracic esophagus. Surgery 141: 570-580, 2007.

20 McGuill MJ, Byrne P, Ravi N and Reynolds J: The prognostic impact of occult lymph node metastasis in cancer of the esophagus or esophago-gastric junction: systematic review and meta-analysis. Dis Esophagus 21: 236-240, 2008.

21 Komukai S, Nishimaki T, Suzuki T, Kanda T, Kuwabara S and Hatakeyama K: Significance of immunohistochemical nodal micrometastasis as a prognostic indicator in potentially curable oesophageal carcinoma. Br J Surg 89: 213-219, 2002.

22 Kinjo T, Shimoji H, Nagahama M, Karimata H, Yoshimi N and Nishimaki T: Prognostic significance of simultaneous presence of histological and immunohistochemical metastasis to lymph nodes in patients with esophageal cancer. Ann Thorac Cardiovasc Surg 20: 951-960, 2014

23 Doki Y, Ishikawa O, Mano M, Hiratsuka M, Sasaki Y, Kameyama M, Ohigashi H, Murata K, Yamada T, Miyashiro I, Yokoyama S, Ishiguro S and Imaoka S: Cytokeratin deposits in lymph nodes show distinct clinical significance from lymph node micrometastasis in human esophageal cancers. J Surg Res 107: 75-81, 2002.
Received October 19, 2017

Revised December 4, 2017

Accepted December 7, 2017 